\title{
Beyond data, innovation, social network, and convergence
}

\author{
Chung Joo Chung ${ }^{1}$ Han Woo Park ${ }^{2}$ (D)
}

Published online: 12 December 2017

(C) Springer Science+Business Media B.V., part of Springer Nature 2017

Since the World Association for Triple Helix and Future Strategy Studies (WATEF) launched DISC (Daegu-Gyeongbuk International Social Network Conference) in 2013, DISC, now known as Data, Innovation, Social Network, and Convergence as four vectors into the next generation in research, has become a faithful and energetic concourse for collaboration and networking among scholars, industry leaders, and public sector experts from various fields all over the world.

The DISC in December 2016, endorsed by INSNA (International Network for Social Network Analysis), was the fourth annual conference hosted in Daegu, Korea. Jointly with CeDEM (Conference for E-Democracy and Open Government) ASIA 2016, the academic festival specifically attracted hundreds of government officials from Asian countries, leading scholars, CEOs, practitioners, and policy-makers using open data. The DISC 2016 focused on network science and communication, new knowledge creation for technological change, creativity and innovation, data-driven marketing and artificial intelligence, entrepreneurial university metrics, and e-democracy and government, which create opportunities for academics to test theories and revise methodology, respectively.

The integration of network science and communication research provides a unique perspective for understanding social structures that shape human and organizational behaviors. Networks can be found everywhere, from the Internet to social networks (Barabási 2016). Network insights reveal how individuals seek social capital and how

Han Woo Park

hanpark@ynu.ac.kr

Chung Joo Chung

cjchung@knu.ac.kr

1 Department of Journalism and Mass Communication, Kyungpook National University, Daegu, South Korea

2 Department of Media and Communication, Interdisciplinary Program of Digital Convergence Business, and Cyber Emotions Research Institute, YeungNam University, Gyeongsan, South Korea 
embedded social reality shapes the perception of intimacy and trust. Network science is also key in tracking how information flows and innovation emerges through behavioral modeling and word-of-mouth. Beyond individual behaviors, organizations are also constrained and supported by a networked social reality. As the development of computational techniques allows us to extract increasingly large networks, it is critical to interpret meanings and messages behind various network shapes and outcomes.

Considering that diffusion is the process in which an innovation is communicated between the past and the present and through which an innovative idea, product, technology, process, or service is adopted (Rice 2017), it is also important to link prior findings and theories in knowledge creation and knowledge diffusion research to new analytics. In this regard, it must be noted that the Journal of Contemporary Eastern Asia (Vol. 16, No. 1, https://jceasia.org) published a sister Special Issue on the DISC 2016. In recent years, knowledge creation and diffusion between universities, firms, and communities have led to technological progress, economic development, and social innovation during dynamic changes and industry transitions. The focal research point of knowledge creation and diffusion involves the specific process of how an individual, a group, an organization, and a country engage in continuous knowledge creation and diffusion regimes while establishing absorptive capacities.

The understanding of such phenomena and hidden processes can be extended and investigated not only through the existing pervasive research approach, such as quantitative methodologies based on patent and bibliography data, but also through qualitative research methodologies, such as interviews and case studies. In particular, big data and its environment enable us to consider new approaches to understanding innovation activities and new phenomena.

Artificial Intelligence (AI) is a notable phenomenon embracing recent trends and determining the evolution of knowledge and systems. The worldwide presence of AI needs to be quantified (Omar et al. 2017). Artificial intelligence plays a significant role in efficiently and effectively exploring the richness of big data and increasing sales. In particular, data scientists from industry and research institutions in the era of artificial intelligence show the impacts of advanced machine learning on the evolution of social science theory.

Metrics are increasingly influential, especially in decision-making in government, industry, and academia. The combination of competitive elements and transparency has created a new and powerful social-communications paradigm, with rankings, reputation, and reality all influencing each other to various degrees. With the growth of open big data, the creation of indicators has been democratized. Accordingly, democratization necessitates a discussion of the ethics, design principles, and use of such indicators.

While big data has come to dominate the lexicon in marketing and the business landscape, new industries are explored by entrepreneurs; this not only opens up new ways to mine the Web and social media for consumers and businesses, but also creates challenges for industry to derive real business value from these activities. The job of data-driven marketers is to extract meaningful messages for the target audience at the right time, and thus stimulate desired consumer behavior.

The power of the Web and social media also leads to challenge collective action and promotes both e-democracy and e-participation around the world. Beyond Government 2.0 with social media, nonhierarchical, nonlinear, and multi-channel connectivity between citizens and governments (Jun and Chung 2016) undoubtedly poses a set of critical challenges and opportunities related to civic engagement and collaborative governance. Furthermore, the growing momentum behind the open data movement, pushing for greater transparency and increased citizen participation in public affairs, has inspired 
policymakers, activists, researchers, and technologists to propose new frameworks for sustainable governance.

There have been several attempts to encompass and explore vastly different disciplines and cultures beyond traditional concepts and approaches in academia. This special issue aims to demonstrate academic efforts to overcome theoretical and methodological limitations in data, innovation, social network, and convergence research.

Jungwon Yeo and her colleagues deal with the nuances of general culture involved in the formation and differentiation of risk communication. Using an innovative method to examine cultural tendencies embedded in the 2016 Louisiana flood response on Twitter, they highlight strong cultural characteristics of the flood response communication, revealing the notions of Louisiana - faith-based, local authority, and nonprofit organizations. They identify cultural similarities in both intra-/inter-group response communication sharing a common cultural background and language use.

Brian Williams and his colleagues explore the relationship between trust in an organization and an individual's propensity to use social media during a disaster. They investigate whether the source of trustworthy information explains variation in the use of social media as an avenue of communication using data from a 2012 survey conducted by the American Red Cross. They show that ability of local governments to get the public to comply with protective orders through social media will increase with public trust, and that making social network systems, with coordination from non-governmental organizations, a part of everyday life can positively affect disaster operations.

Using emergency medicine surveys in Korea, Minsun Song investigates the effects of a strong commitment driven by exercises on building sustainable emergency management networks. She reviews the mechanism, coordination, division, and defection problems in collaboration risk in response to the collective action dilemma and strong commitment driven by repetitive drills as a means of overcoming collective action dilemmas. She emphasizes that the belief in the good operation of a network is related to the level of trust among organizations.

Vargas Meza Xanat and her colleague analyze the international trade network of key commodities such as grains and other GMO-related products over nearly three decades. They discuss interesting tendencies in global trade policies and the economic implications of results for specific countries. They indicate that trade in agricultural commodities has become more diversified over time.

In the context of the new public management regime-based higher education reform, Joonha Jeon and So Young Kim examine whether inequality between universities is increasing in terms of research output. Using a novel dataset containing all universities in Korea over seven years, they draw a map for exploratory analysis of the longitudinal trends of inequality in university research output. They argue that the perceived type of inequality among the key stakeholders in the higher education sector in a given policy context is closer to the absolute inequality.

Meijun Liu and her colleagues investigate the disciplinary structure and its evolution in knowledge absorption of the Humanities and Social Sciences in China. Based on the data of Chinese and English articles published by researchers in China from 1998 to 2014, they explore the knowledge flow in the field. As the very first systematic study on disciplinary knowledge flow in China, they capture the dynamic process of knowledge absorption, diffusion, and reproduction in the Humanities and Social Sciences in China.

Seyed Jalali and Han Woo Park explore the most recent stage in the scholarly development of Business Analytics. Using research publications collected from the Web of Science, they explain a thematic cluster focusing on analytical techniques and tools 
particular to the rapid dissemination of big data-related studies. It is noteworthy that the research shows the important role of data mining and its applications, and visualizes the flow of collaborations among countries in the area.

By analyzing critical stakeholders and keywords embedded in self-organizing networks on social media, Eun Sun Lee and Kyujin Jung explore the ecology of the social economy. They investigate the evolution of the recent Facebook discourse on the social economy in Korea. They try to provide policy implications for Asian countries with underdeveloped social economies and theoretically contribute to the field of public policy analysis and management.

Young-Joo Lee and Ji-Young Park try to foresee the advent of new technologies and their socio-economic impacts by identifying emerging issues based on the weak signal detection approach. They propose a framework that incorporates quantitative and qualitative text mining for assessing the strength of future signals. Then, they apply the framework to the case study of EBSCO data on the ethical issues of artificial intelligence and provide an improved methodology to verify the reliability and validity of analytical results by combining qualitative and quantitative analysis methods.

The editors first thank the authors who contributed to this special issue for their commitment to studying data, innovation, social network, and convergence. The editors are also grateful to the anonymous reviewers for their valuable comments and suggestions, which have been vital in improving the quality of papers in the special issue. Finally, Vittorio Capecchi, the Editor-In-Chief, deserves special recognition for his support and guidance in compiling this special issue in Quality \& Quantity.

\section{References}

Barabási, A.-L.: Network Science. Cambridge University Press, Cambridge (2016)

Jun, C., Chung, C.: Big data analysis of local government 3.0: Focusing on Gyeongsanbuk-do in Korea. Technol Forecast Soc 110, 3-12 (2016)

Omar, M., Mehmood, A., Choi, G.S., Park, H.W.: Global mapping of artificial intelligence in Google and Google Scholar. Scientometrics 113, 1269-1305 (2017)

Rice, R.E.: Intermediality and the diffusion of innovations. Hum Commun Res 43, 531-544 (2017) 